\title{
mm-Wellen RFID
}

\section{Design und Implementierung eines Demonstrators}

\author{
P. F. Freidl, M. E. Gadringer, S. W. Sattler, D. Amschl, B. Auinger, G. Holweg, U. Mühlmann, W. Bösch
}

\begin{abstract}
Mit der zunehmenden Verbreitung des Internets der Dinge steigt auch der Bedarf an miniaturisierten, stromsparenden und drahtlos angebundenen Sensoren und Geräten. Eine Möglichkeit, diese Anbindung zu realisieren, ist die Übertragung des UHF RFID Konzeptes der Rückstrahlkommunikation in den mm-Wellen Frequenzbereich. Die dadurch erreichbare Verkleinerung der Sensoren und die in diesen Frequenzbereichen verfügbaren Bandbreiten ermöglichen eine Vielzahl neuer Anwendungen. In diesem Artikel werden das Design und die Implementierung eines Hardware-Demonstrators vorgestellt, welcher nicht nur die Machbarkeit einer solchen Kommunikation bei mm-Wellen zeigt, sondern auch die Funktionalität des EPC Gen2 Protokolls unter den vorgestellten Bedingungen umsetzt.
\end{abstract}

Schlüsselwörter: mm-Wellen RFID; MMID; Rückstrahlkommunikation; Internet der Dinge; loT

mm-wave RFID. Design and implementation of a demonstrator.

The Internet of Things (IOT) and its applications demand for solutions for small and low-power communication devices. Overcoming the drawbacks of UHF radio frequency identification (RFID) a shift of the backscatter communication principle to mm-wave frequencies is desirable. With the significant reduction of the transponder size and wider communication bandwidth, many new applications become possible. Demonstrating the feasibility, we implemented a fully functional MMID system in the E-band. Both, the base station and the transponder are investigated and their performance is presented in a system context.

Keywords: mm-wave RFID; MMID; backscatter communication; Internet of Things; loT; E-band

Eingegangen am 6. Februar 2016, angenommen am 14. April 2016, online publiziert am 25. Mai 2016 (C) The Author(s) 2016. Dieser Artikel ist auf Springerlink.com mit Open Access verfügbar

\section{Einleitung}

Unter dem Schlagwort "Internet der Dinge" (Internet of Things, IoT) versteht man eine immer engere Anbindung von Geräten und Sensoren an ein globales Netzwerk. Diese Anbindung birgt ein erhebliches Potential für neue Anwendungen in vielen Bereichen der Wirtschaft und Industrie, wie Produktion, Logistik oder Gesundheitswesen, sowie auch für den täglichen Gebrauch des privaten Anwenders. Es wird geschätzt, dass sich bis 202026 Milliarden Geräte mit einem loT Kontext im Umlaufen befinden werden [1].

Viele dieser Anwendungen benötigen Sensoren, die drahtlos an eine Basisstation angebunden werden. Um einen vielseitigen Einsatz der Sensoren zu erzielen, ist eine möglichst kleine Baugröße von entscheidendem Vorteil. Zudem benötigen die Sensoren meist keine hohen Datenraten, müssen aber einen sehr geringen Stromverbrauch aufweisen, um einen dauerhaften als auch wartungsarmen Betrieb sicher zu stellen [2].

Eine Technologie, welche diese Anforderungen erfültt, ist UHF RFID [3]. Bei dieser Technologie wird der Transponder (Sensor) von der Basisstation mittels elektromagnetischen Wellen mit Energie versorgt. Der Sensor nutzt diese Wellen nicht nur zur Stromversorgung, sondern moduliert außerdem seine Daten auf den Träger der Basisstation mittels Rückstreumodulation auf [4]. Diese Art der Modulation ermöglicht eine äußerst stromsparende Implementierung des Senders im Transponder. Der Nachteil an derzeit kommerziell verfügbaren RFID Systemen ist die Größe der Sensorantenne die, aufgrund der gewählten Trägerfrequenz, einen Großteil der Sensorgröße ausmacht. Zudem führt die steigende Nutzung dieser UHF Frequenzbänder zu Interferenzproblemen. Außerdem würde eine Erhöhung der nutzbaren Kanalbandbreite Anwendungen, wie zum Beispiel die Lokalisierung des Transponders, erheblich verbessern.

Diese Nachteile werden durch eine substanzielle Erhöhung der Trägerfrequenz in den mm-Wellen Bereich beseitigt. Die Umsetzung von RFID bei mm-Wellen wurde in der Literatur unter dem Schlagwort MMID (mm-Wellen Identifikation) vorgestellt [5]. Die höhere Trägerfrequenz erlaubt eine signifikante Reduktion der Antennengröße unter Beibehaltung deren Effizienz. Die dadurch erreichbare Verkleinerung der Abmessungen ermöglicht sogar die Integration der Antenne in den Chip des Transponders oder dessen Gehäuses. Zusätzlich sind bei diesen Frequenzen die verfügbaren Bandbreiten weitaus größer und ermöglichen daher Anwendungen wie Lokalisierung [6]. Die Erhöhung der Trägerfrequenz bringt aber auch Nach-

Freidl, Philipp Franz, Institut für Hochfrequenztechnik, TU Graz, Inffeldgasse 12, 8010 Graz, Österreich and Development Center Graz, Infineon Technologies Austria AG, Babenbergerstrasse 10, $8020 \mathrm{Graz}$, Österreich

(E-Mail: philipp.freidl@tugraz.at; philipp.freidl@infineon.com); Gadringer, Michael Ernst, Institut für Hochfrequenztechnik, TU Graz, Inffeldgasse 12, 8010 Graz, Österreich (E-Mail: michael.gadringer@tugraz.at); Sattler, Sebastian Wolfgang, Institut für Hochfrequenztechnik, TU Graz, Inffeldgasse 12, 8010 Graz, Österreich; Amschl, Dominik, Institut für Hochfrequenztechnik, TU Graz, Inffeldgasse 12, $8010 \mathrm{Graz}_{\text {。 }}$ Österreich; Auinger, Bernhard, Institut für Elektronik, TU Graz, Inffeldgasse 12 , 8010 Graz, Österreich (E-Mail: bernhard.auinger@tugraz.at); Holweg, Gerald, Development Center Graz, Infineon Technologies Austria AG, Babenbergerstrasse 10, 8020 Graz, Österreich (E-Mail: gerald.holweg@infineon.com); Mühlmann, Ulrich, NXP Semiconductors, Mikron-Weg 1, 8101 Gratkorn, Österreich

(E-Mail: ulrich.muehlmann@nxp.com); Bösch, Wolfgang, Institut für Hochfrequenztechnik, TU Graz, Inffeldgasse 12, 8010 Graz, Österreich (E-Mail:wbosch@tugraz.at) 
teile mit sich. Vor allem die hohe Dämpfung in der drahtlosen Übertragung reduziert die Reichweite solcher Systeme enorm [7].

In der Literatur wurden bereits Konzepte vorgestellt, welche die Funktionalität von einzelnen Komponenten eines MMID Systems demonstrieren. In [8-10] zeigen die Autoren die Umsetzbarkeit der Rückstrahlmodulation und der Hüllkurvendetektion für mm-Wellen Transponder. Ein voll funktionales EPC Gen2 [11] kompatibles System bei einer Trägerfrequenz von $10 \mathrm{GHz}$, unter Verwendung einer kommerziellen UHF RFID Basisstation und eines UHF RFID Transponder Chips, wurde in [12] beschrieben. Um die Reichweite zu erhöhen, wurde in [13] ein Transponder gezeigt, welcher ein aktives Rückstrahlmodulationsverfahren verwendet. In diesem Fall wird der Träger mittels einer Verstärkerschleife regeneriert. Um die Machbarkeit einer vollständigen Integration eines MMID Transponders auf einen einzelnen Chip zu zeigen, wurde in [14] die Implementierung von mm-Wellen Antennen auf einem CMOS Prozess untersucht. Weitere Publikationen [15-18] zeigen die Implementation von Teilkomponenten aktiver Transponder auf integrierten Schaltungen, bei welchen das Sendesignal vom Transponder selbst generiert wird.

In diesem Artikel präsentieren wir einen Demonstrator für ein vollständiges MMID System, welcher die Funktionalität des EPC Gen2 Protokolls von UHF RFID bei Trägerfrequenzen jenseits von $70 \mathrm{GHz}$ implementiert.

\section{System-Konzept}

Der MMID Demonstrator besteht aus einer "Software Defined Radio" (SDR) Basisstation und einem batteriegespeisten Transponder (Abb. 1). Wie bei RFID Systemen üblich, sendet die Basisstation nicht nur Daten oder Kommandos an den Transponder, sondern stellt auch den Dauerstrichton für die Antwort des Transponders bereit.

\subsection{Basisstation}

Die Basisstation des MMID Systems besteht aus zwei Hauptteilen: einem direkt umsetzenden $\mathrm{mm}$-Wellen Sendeempfänger und einer SDR Plattform. Abbildung 2 zeigt das Konzept der Basisstation. Die SDR Plattform setzt sich aus einem Digital/Analog (D/A) Umsetzer, einem Zwei-Kanal Zwischenfrequenzverstärker und einem Analog/Digital Umsetzer zusammen, welche von einem MATLAB Programm auf einem Hostrechner gesteuert werden. Im Sendefall erstellt das MATLAB Programm eine EPC Gen2 Anfrage, welche auf einen $10 \mathrm{MHz}$ Träger aufmoduliert ist. Dieses komplexwertige Basisbandsignal wird dann über den D/A Umsetzer an den Sendeempfänger weitergegeben. Im Empfangsfall kommt die ebenfalls auf einen $10 \mathrm{MHz}$ Träger aufmodulierte Antwort des Transponders vom Sendeempfänger. Diese wird mittels des variablen Basisbandverstär-

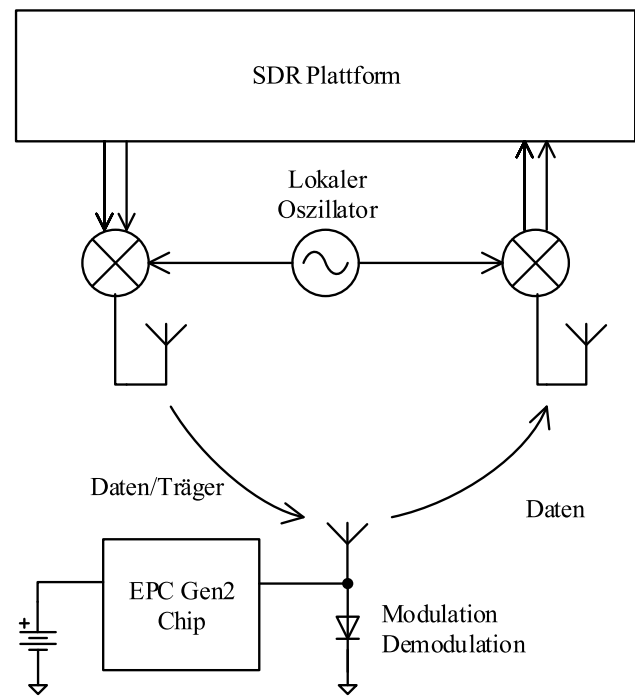

Abb. 1. Konzept des mm-Wellen RFID Systems mit einer SDR Plattform und einem batterieunterstützten Transponder

kers an den Eingang des A/D Umsetzers angepasst und anschließend digitalisiert. Das MATLAB Programm übernimmt dann die Übersetzung des komplexen Empfangssignal in einen binären Datenstrom.

Der mm-Wellen Sendeempfänger setzt die Sende- und Empfangssignal direkt auf eine Mittenfrequenz von $72,5 \mathrm{GHz}$, beziehungsweise in das Basisband um. Diese Frequenz wurde im Gegensatz zu dem im mm-Wellenbereich verfügbaren $60 \mathrm{GHz}$ ISM Band gewählt, da in diesem Bereich die verfügbaren Komponenten der Basisstation und des Transponders am Besten zusammenspielen. Das so generierte Sendesignal wird dann über eine E-Band Hornantenne mit $21 \mathrm{~dB}$ Gewinn an den Transponder übertragen. Nach dem Senden der EPC Gen2 Anfrage gibt der Sendeempfänger weiterhin einen Dauerton auf seiner Mittenfrequenz aus, um so dem Transponder den benötigte Träger zur Rückstrahlkommunikation zu Verfügung zu stellen. Im Empfangspfad nimmt der mm-Wellen Sendeempfänger die vom Transponder generierte Antwort über eine weitere Hornantenne auf. Nach Umsetzung ins Basisband wird die Rückantwort von dem ZF Verstärker verstärkt und am Eingang des Analog/Digital (A/D) Umsetzers zur Verfügung gestellt. Die Trennung von Sende- und Empfangsantenne hat den Vorteil eines weit geringeren Übersprechens zwischen dem Sende- und Empfangssignal und verbessert daher die Leistungsfähigkeit des Empfängers signifikant. Abbildung 3 zeigt eine Fotografie der Basisstation, auf der die einzelnen Komponenten ersichtlich sind.

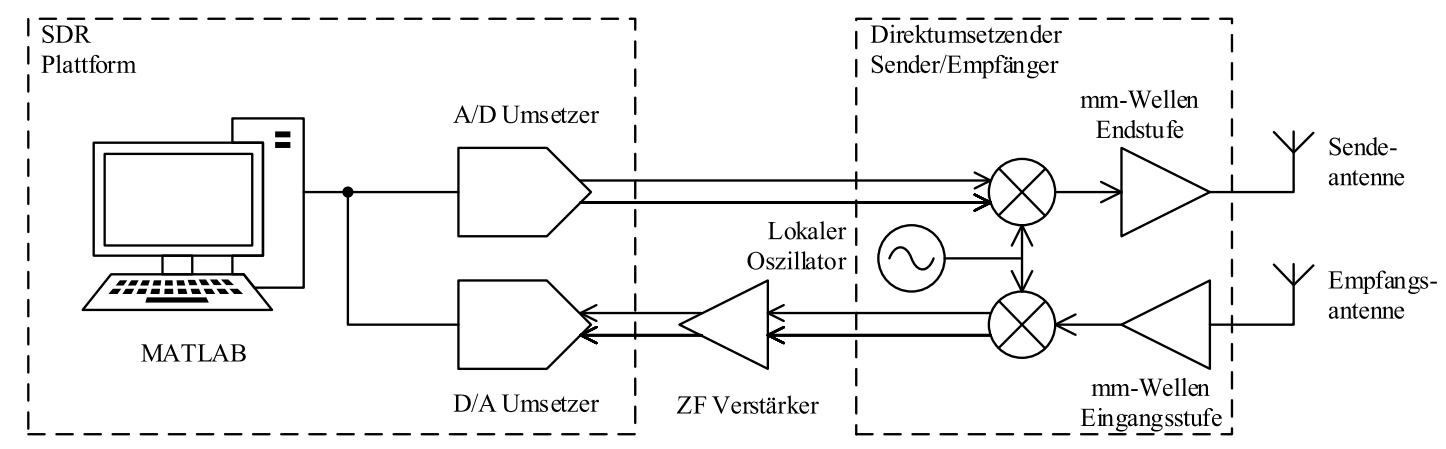

Abb. 2. Konzept der MMID Basisstation mit der SDR Plattform, und dem mm-Wellen Sender/Empfänger 


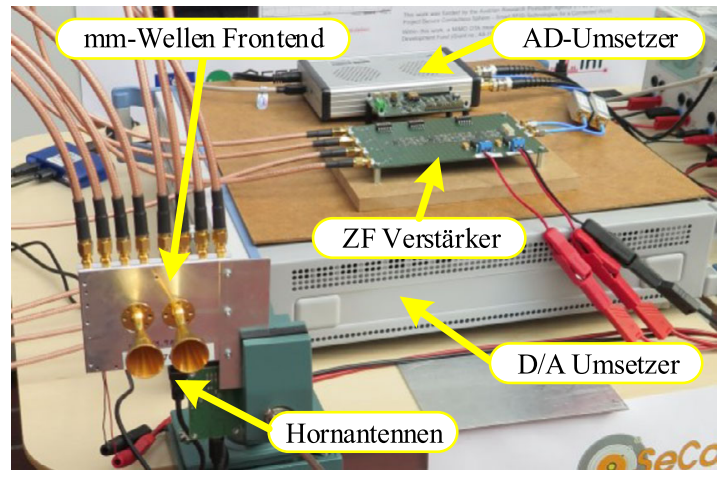

Abb. 3. Fotografie der Basisstation

\subsection{Transponder}

Um ein flexibles Design zu ermöglichen, besteht der Transponder des MMID Systems aus zwei Hauptkomponenten: einer Basisbandeinheit und einem mm-Wellen Frontend. Die Basisbandeinheit basiert auf einem Entwicklungschip der Firma NXP Semiconductors, welcher den EPC Gen2 Protokollstack für den Transponder implementiert. Das mm-Wellen Frontend ist als separate Platine ausgeführt, welche auf die Hauptplatine mit der Basisbandeinheit aufgesteckt wird, und beherbergt als zentrales Element eine mm-Wellen Schottkydiode von Infineon Technologies (BAT14-077D, [19]).

Der EPC Gen2 Chip der Basisbandeinheit implementiert die Basisbandfunktionalität eines EPC Gen2 Transponders und verfügt über ein Ein-Pin Interface, welches für TTL Pegel ausgelegt ist. Um mit diesem Interface kommunizieren zu können, ist auf der Basisbandeinheit eine Pegelanpassung und eine Logik zur Umschaltung zwischen dem Empfangs- und Sendemodus des Transponders untergebracht. Im Empfangsfall wandelt diese Schaltung das kleine Signal der mmWellen Diode in TTL Signale um und sendet diese an den Chip. Im Sendefall generiert die Schaltung aus der Antwort des Chips zwei unterschiedliche Biasspannungen für die mm-Wellen Diode, um so die Rückstrahlmodulation zu erzeugen (Abb. 4).

Auf der mm-Wellen Platine ist neben der Diode eine Antenne, ein Anpassnetzwerk zwischen der Diode und der Antenne, sowie ein Tiefpassfilter untergebracht. Die mm-Wellen Antenne ist als einzelne Mikrostreifenantenne mit $7 \mathrm{dBi}$ Gewinn ausgeführt. Dies ermöglicht neben einer sehr geringen Größe von ungefähr $1 \times 1 \mathrm{~mm}$ eine relative robuste Kommunikation mit der Basisstation, da die Hauptkeule der Antenne eine Breite von 75 Grad aufweist. Im Empfangsmodus des Transponders wird die mm-Wellen Diode als Hüllkurvendetektor verwendet. Im Sendemodus wird die Impedanz der Diode

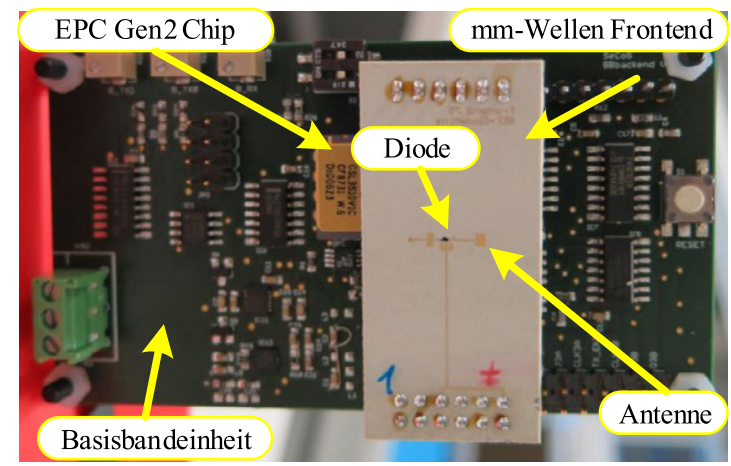

Abb. 5. Fotografie des Transponders

mittels verschiedener Biasspannungen variiert. Dies führt zu einer Änderung des Reflektionsfaktor am Fußpunkt der Antenne somit zu einer Beeinflussung des Pegel des in Richtung der Basisstation zurückgesendete Dauerstrichtons. Auf diese Weise werden die Daten des Transponders auf den Dauerstrichton aufmoduliert. Die Biasspannungen werden sowohl im Empfangs- als auch im Sendemodus über einen 2,5 k $\Omega$ Widerstand der Diode zugeführt.

Abbildung 5 zeigt eine Fotografie des Transponders mit der mmWellenplatine, welche auf das Basisbandboard aufgesteckt ist. Um die TTL Chips mit Energie zu versorgen und der mm-Wellen Diode die entsprechenden Biasströme zu Verfügung zu stellen, wird der Transponder mit einer Batterie gespeist.

\section{Messungen}

Um die Funktionalität und Leistungsfähigkeit des MMID Systems zu verifizieren, wurden verschiedene Messungen an der Basisstation, dem Transponder als auch dem Gesamtsystem durchgeführt. Für die Systemmessungen wurde der Transponder ungefähr $10 \mathrm{~cm}$ von der Basisstation entfernt aufgestellt. Die Basisstation generiert einen EPC Gen2 Request Befehl, der alle 10 ms wiederholt wird.

Im ersten Schritt wurde der Hüllkurvendetektor des mm-Wellen Transponder-Frontends evaluiert. Hierzu wurde dieses mit einem Oszilloskop mittels AC-Kopplung verbunden und über einen $2.5 \mathrm{k} \Omega$ Widerstand mit einem Biasstrom beaufschlagt (Abb. 10). Wie aus Abb. 6 zu entnehmen ist, zeigt das gemessene Hüllkurvensignal am Ausgang des Tiefpassfilters eine Amplitude von $8 \mathrm{mV}$. Zusätzlich wurde die empfangene Leistung am Antennenfußpunkt mittels eines Spektrumanalyzers im Zero-Span Modus bei der Trägerfrequenz gemessen. Dieses weist eine Modulationstiefe von etwas mehr als $30 \mathrm{~dB}$ auf.

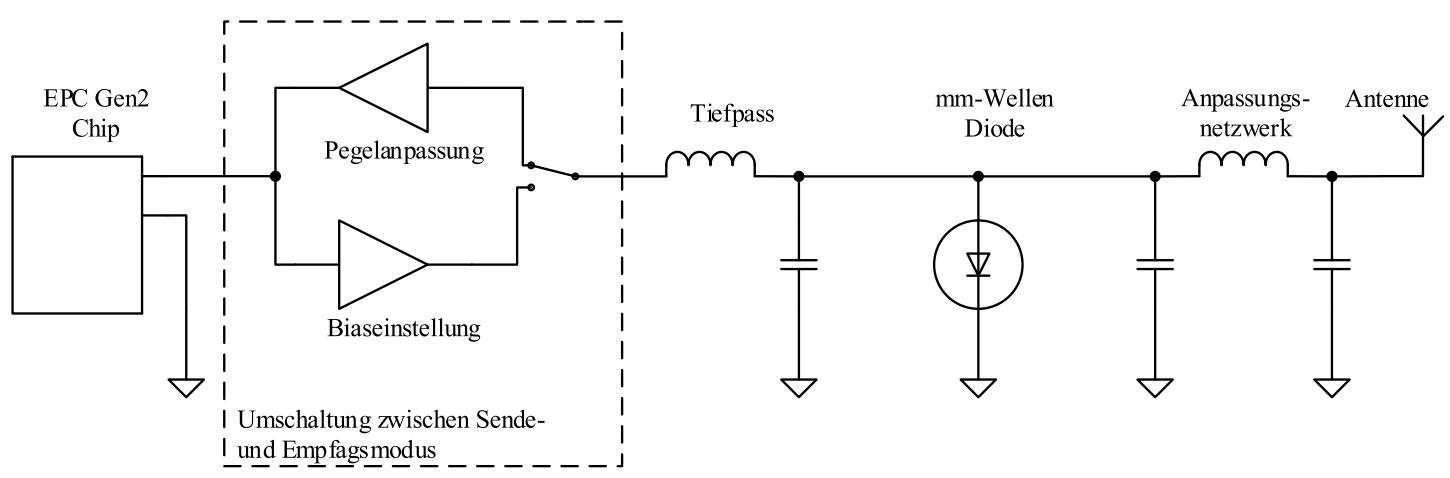

Abb. 4. Konzept des MMID Transponders 

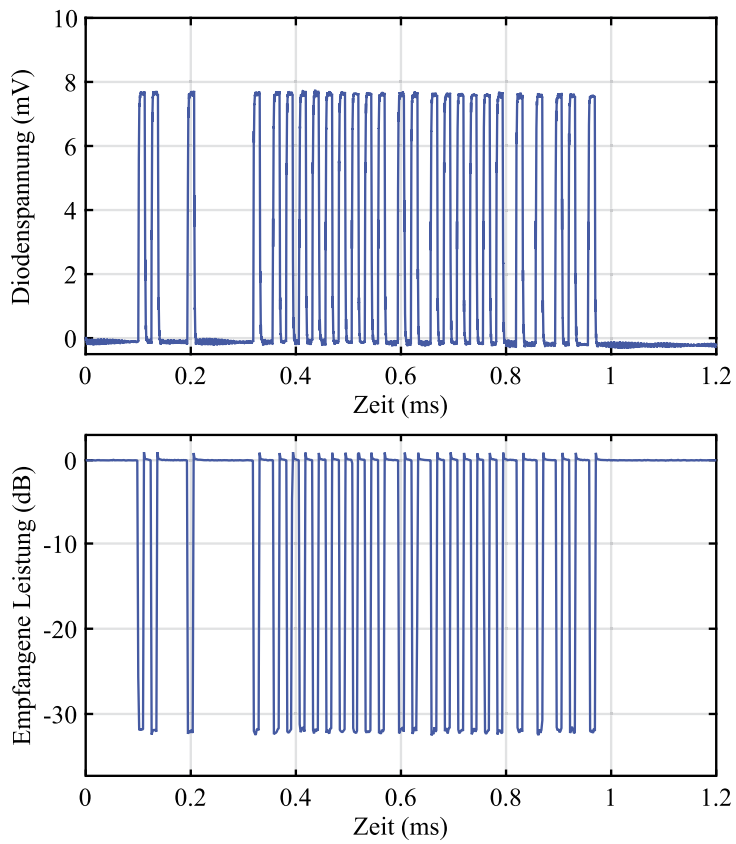

Abb. 6. Spannung an der Diode über den Lastwiderstand (oben) und die relative empfangene Leistung bei $72.5 \mathrm{GHz}$ (unten)

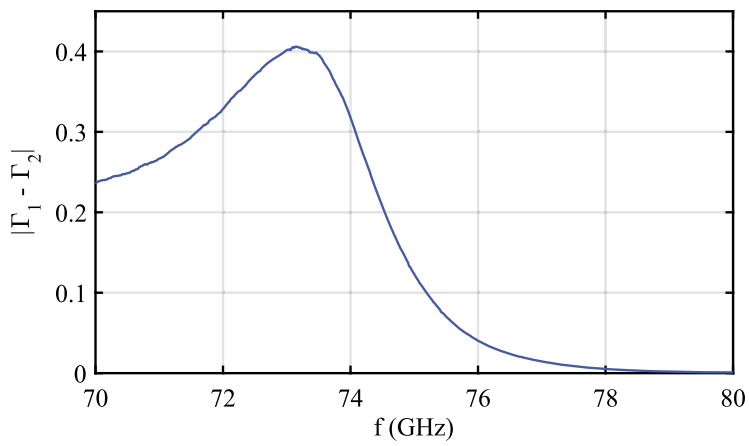

Abb. 7. Modulationstiefe des Transponders

In einem weiteren Schritt wurde die Modulationsfähigkeit des Transponders getestet. Um dies zu bewerkstelligen, wurde ein $\mathrm{mm}$ Wellen Frontend gefertigt, bei welchem anstatt der Antenne ein Testport platziert wurde. Dieses modifizierte Frontend wurde mit einem Netzwerkanalysator verbunden, um den Eingangsreflektionsfaktor bei zwei verschiedenen Biasströmen $\left(\Gamma_{1}\right.$ und $\left.\Gamma_{2}\right)$ zu bestimmen. Die Differenz dieser beiden Reflektionsfaktoren ist in Abb. 7 zu sehen. Sie beträgt für die gewünschte Trägerfrequenz von $72,5 \mathrm{GHz}$ ungefähr 0,35 und steigt maximal auf 0,4 bei $73 \mathrm{GHz}$. Verglichen mit bereits publizierten Transpondern für MMID [9] stellt dies eine Verbesserung um bis zu $50 \%$ dar.

Nach der Verarbeitung des von der Diode demodulierten Hüllkurvensignals durch den EPC Gen2 Basisbandchip, sendet dieser seine Antwort durch Modulation des Biasstroms der Diode an die Basisstation zurück. Diese transformiert das Signal aus dem mm-Wellen Bereich auf eine Zwischenfrequenz von $10 \mathrm{MHz}$. Abbildung 8 zeigt das normierte Amplitudenspektrum des digitalisierten Signals der Basisstation. Es zeigt die beiden Subträger des rückstrahlmodulierten Signals mit 160 kHz Abstand zum Träger. Das Verhältnis zwischen der Amplitude des Trägers und der des Subträgers beträgt $-38 \mathrm{dBc}$. Zusätzlich weist der informationshaltige Subträger einen

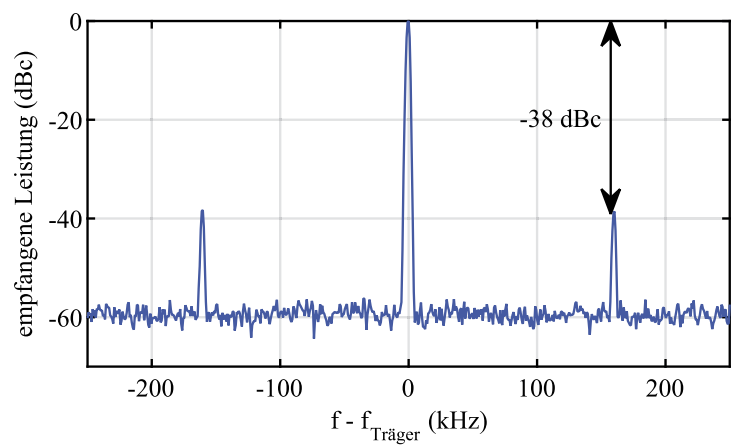

Abb. 8. Spektrum des an der Basisstation gemessenen Antwort des Transponders

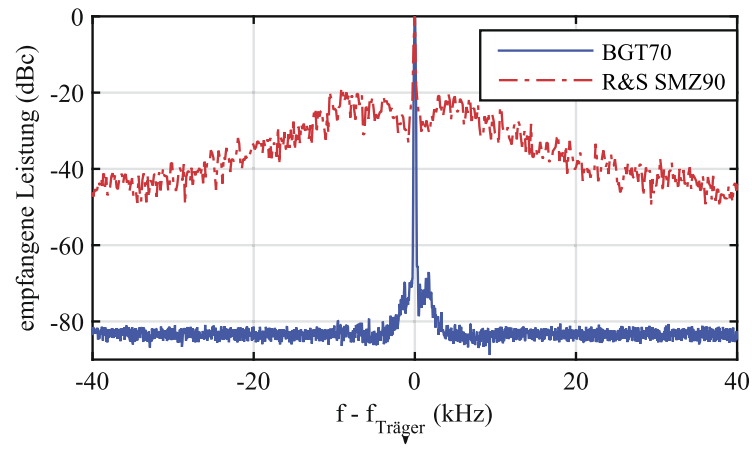

Abb. 9. Phasenrauschen am Empfänger der Basisstation

Signalrauschabstand von $20 \mathrm{~dB}$ auf. Diese Werte zeigen eindeutig, dass die Sensitivität des Empfängers für die Demodulation des Transpondersignals ausreicht, und die maximale Reichweite der Basisstation noch nicht ausgereizt ist.

Für die Leistungsfähigkeit des MMID Systems ist das Phasenrauschen des mm-Wellen Senders/Empfängers der Basisstation ein entscheidender Faktor [17]. Zur Quantifizierung des Phasenrauschens des mm-Wellen Empfängers wurde ein Dauerstrichton mittels eines hochstabilen Signalgenerators (Rhode\&Schwarz SMZ90 in Kombination mit einem Rhode\&Schwarz SMF100A) in $10 \mathrm{MHz}$ Abstand zur Trägerfrequenz generiert. Dieser wurde vom Empfänger in die Zwischenfrequenz übersetzt und dort mittels eines Spektrumanalysators aufgezeichnet. Wie in Abb. 9 zu sehen ist, überlagert sich das Phasenrauschen des Empfängers mit dem Eingangssignal und resultiert in einem Grundrauschen welches nur ca. ( $-20 \mathrm{dBc}$ im Abstand von $10 \mathrm{kHz}$ ) aufweist. In diesem Betriebsmodus ist die Sensitivität der Basisstation signifikant eingeschränkt.

Das hohe Phasenrauschen des mm-Wellen Empfängers kann durch die Generierung des lokalen Oszillators durch eine Frequenzmultiplikation eines PLL-Signals mit dem Faktor 64 erklärt werden [20]. Betrachtet man jedoch das Phasenrauschen unter Verwendung eines Dauerstrichtons welcher mittels des mm-Wellen Senders der Basisstation generiert wurde, zeigt sich, dass sich dieses deutlich reduziert und für die Leistungsfähigkeit des Empfängers vernachlässigt werden kann. Diese signifikante Verbesserung kann durch die Verwendung von ein und demselben lokalen Oszillator für den Sendeals auch den Empfangspfad der Basisstation erklärt werden. Solange die Laufzeit des Trägers - von der Basisstation zum Transponder und zurück - kürzer ist als die Kohärenzzeit des Phasenrauschens, wird dieses im Mischprozess des Empfängers kompensiert. Erst dieser Effekt ermöglicht die hohe Sensitivität der Basisstation und damit einen erfolgreichen Betrieb des MMID Systems. 


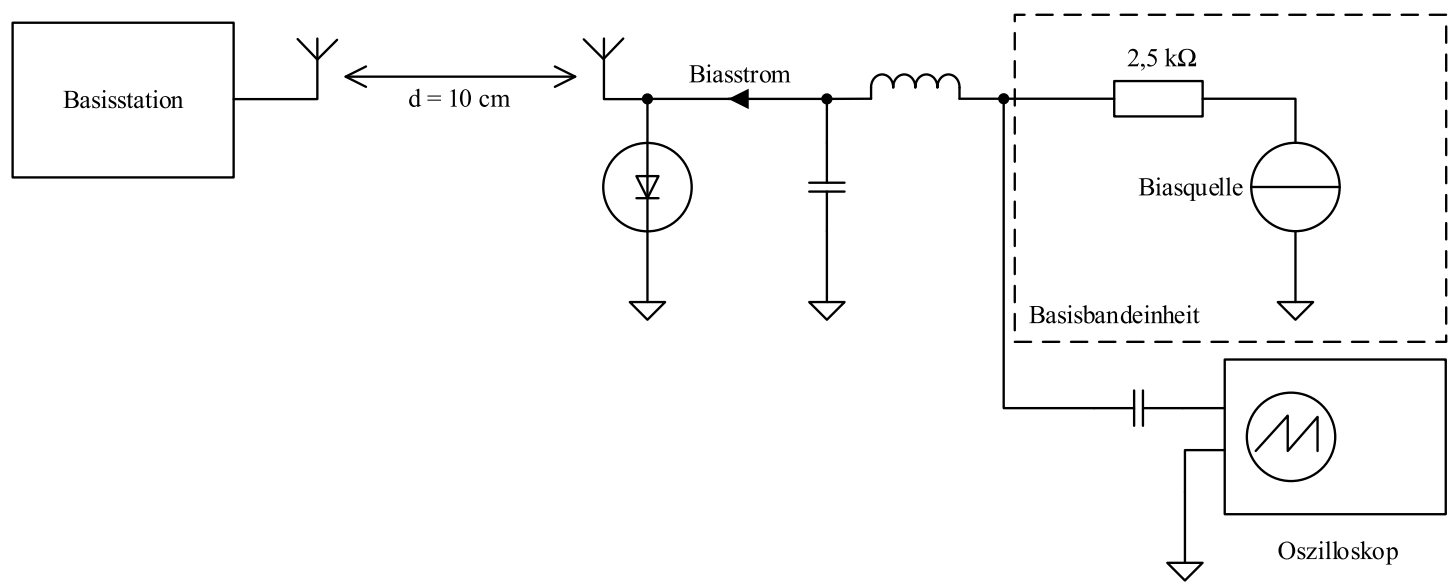

Abb. 10. Aufbau der Messung der Demodulationseigenschaften des Transponders.

\section{Conclusio}

In diesem Artikel wurde ein mm-Wellen RFID System gezeigt, welches die volle Funktionalität des EPC Gen2 Standards abbildet. Das Systemkonzept mit dem Design von Basisstation sowie Transponder wurde ausführlich diskutiert und beschrieben. Die Funktionalität des Systems wurde anhand von Messungen bei einem Abstand von $10 \mathrm{~cm}$ zwischen der Basisstation und dem Transponder untersucht. Im Zuge der Messungen hat sich gezeigt, dass die Verwendung von einem einzigen lokalen Oszillator für den Sende- und Empfangspfad der Basisstation für die Funktionalität des Gesamtsystems von entscheidender Bedeutung ist um die Auswirkungen des Phasenrauschens desselben kompensieren zu können.

\section{Danksagung}

Diese Arbeit wurde mit Mitteln der Österreichische Forschungsförderungsgesellschaft FFG im Rahmen des K-Projektes "Secure Contactless Sphere - Smart RFID Technologies for a Connected World" finanziert. Zudem möchten die Autoren Herrn Erich Schlaffer von AT\&S ihren Dank für die Produktion der mm-Wellen PCB aussprechen.

Open Access This article is distributed under the terms of the Creative Commons Attribution 4.0 International License (http://creativecommons.org/ licenses/by/4.0/), which permits unrestricted use, distribution, and reproduction in any medium, provided you give appropriate credit to the original author(s) and the source, provide a link to the Creative Commons license, and indicate if changes were made.

\section{Literatur}

1. Want, R., Schilit, B., Jenson, S. (2015): Enabling the internet of things. Computer, $48(1), 28-35$.

2. DeLisle, J. (2015): 4 major M2M and loT challenges you need to know. Microw. RF, 54(2), 36-38.

3. Finkenzeller, K. (2010): RFID handbook: fundamentals and applications in contactless smart cards, radio frequency identification and near-field communication. 3. Aufl. New York: Wiley.

4. Dobkin, D. M. (2012): The RF in RFID—UHF RFID in practice. 2. Aufl. London: Newnes.
5. Pursula, P., Vaha-Heikkila, T., Muller, A., Neculoiu, D., Konstantinidis, G., Oja, A., Tuovinen, J. (2008): Millimeter-wave identification—a new short-range radio system for low-power high data-rate applications. IEEE Trans. Microw. Theory Tech., 56(10), 2221-2228

6. Pursula, P., Viikari, V., Vähä-Heikkilä, T. (2010): Theory and applications of millimeter wave identification. In 2010 international semiconductor conference, CAS, Oct. 2010 (Bd. 01, S. 53-56).

7. Rappaport, T. S., Heath, R. W. J., Daniels, R. C., Murdock, J. N. (2014): Millimeter wave wireless communications. 1. Aufl. London: Prentice-Hall.

8. Müller, A., Neculoiu, D., Pursula, P., Vaha-Heikkila, T., Giacomozzi, F., Tuovinen, J. (2007): Hybrid integrated micromachined receiver for $77 \mathrm{GHz}$ millimeter wave identification systems. In 10th European microwave conference, Oct. 2007 (S. 1034-1037).

9. Pursula, P., Donzelli, F. (2011): Transponders for millimeter wave identification. In 2011 IEEE-APS topical conference on antennas and propagation in wireless communications, APWC, Sep. 2011 (S. 1221-1224).

10. Kiuru, T., Pursula, P., Rajamaki, J., Vaha-Heikkila, T. (2013): A 60-GHz semipassive MMID transponder for backscattering communications. In 2013 IEEE MTT-s international microwave symposium digest, IMS, Jun. 2013 (S. 1-3).

11. EPC radio-frequency identity protocols generation-2 UHF RFID, GS1 Std.

12. Pursula, P., Donzelli, F., Seppa, H. (2011): Passive RFID at millimeter waves. IEEE Trans. Microw. Theory Tech., 59(8), 2151-2157.

13. Carlowitz, C., Vossiek, M., Strobel, A., Ellinger, F. (2013): Precise ranging and simultaneous high speed data transfer using $\mathrm{mm}$-wave regenerative active backscatter tags. In 2013 IEEE international conference on RFID, RFID, Apr. 2013 (S. 253-260).

14. Fonte, A., Saponara, S., Pinto, G., Neri, B. (2011): Feasibility study and on-chip antenna for fully integrated uRFID tag at $60 \mathrm{GHz}$ in $65 \mathrm{~nm}$ CMOS SOI. In 2011 IEEE international conference on RFID-technologies and applications, RFID-TA, Sept. 2011 (S. 457-462).

15. Pellerano, S., Alvarado, J., Palaskas, Y. (2009): A mm-wave power harvesting RFID tag in $90 \mathrm{~nm}$ CMOS. In IEEE custom integrated circuits conference, Sep. 2009 (S. 677680).

16. Wu, Y., Linnartz, J., Gao, H., Baltus, P., Bergmans, J. (2011): System study of a $60 \mathrm{GHz}$ wireless-powered monolithic sensor system. In 2011 8th international conference on information, communications and signal processing, ICICS, Dec. 2011 (S. 1-5).

17. Burasa, P., Constantin, N., Wu, K. (2014): High-efficiency wideband rectifier for singlechip batteryless active millimeter-wave identification (MMID) tag in 65-nm bulk CMOS technology. IEEE Trans. Microw. Theory Tech., 62(4), 1005-1011.

18. Burasa, P., Constantin, N., Wu, K. (2015): Low-power injection-locked zero-IF selfoscillating mixer for self-powered millimeter-wave identification (MMID) active tag in 65-nm CMOS. In 2015 IEEE radio frequency integrated circuits symposium, RFIC, May 2015 (S. 259-262)

19. Infineon Technologies (2014): Silicon single flip chip Schottky diode BAT14-077D.

20. Infineon Technologies (2014): Single-chip SiGe transceiver chipset for E-band backhaul applications from 71 to $76 \mathrm{GHz}$. 


\section{Autoren}

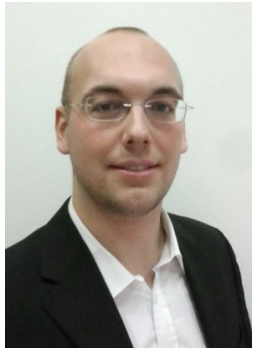

\section{Philipp Franz Freid}

wurde 1987 in Graz geboren. 2013 schloss er an der Technischen Universität Graz das Diplomstudium der Elektrotechnik ab. In seiner Diplomarbeit beschäftigte er sich mit der Entwicklung von Ultra-Breitbandantennen für Kommunikationssysteme in Automobilen. Im Rahmen seiner Dissertation am Institut für Hochfrequenztechnik der TU Graz beschäftigt er sich seit 2013 mit der Entwicklung und Analyse von RFID-Systemen im mm-Wellen-Frequenzbereich. Seit 2016 arbeitet er bei Infineon Technologies Austria AG an der Verifikation und Charakterisierung von mm-Wellen ICs.

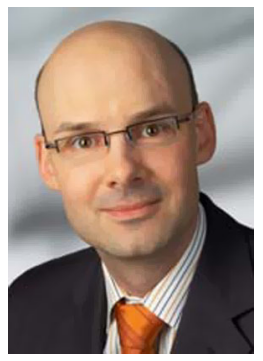

\section{Michael Ernst Gadringer}

erwarb sein Diplom und sein Doktorat im Bereich Nachrichtentechnik an der Technischen Universität Wien in den Jahren 2002 bzw. 2012. Seit 2010 arbeitet er am Institut für Hochfrequenztechnik der Technischen Universität Graz als Universitätsassistent. Während seines Studiums beschäftigte er sich mit dem Design von analogen und digitalen Linearisierungssystemen für Hochfrequenzleistungsverstärker als auch mit nichtlinearer Modelbildung. Weitere Forschungsschwerpunkte bilden die Weiterentwicklung von breitbandigen mm-Wellen-Kommunikationssystemen und deren Messsystemen sowie den dafür benötigten Kalibrations- und Deembedding-Konzepten. Er ist Co-Autor des Buches "RF Power Amplifier Behavioral Modeling", herausgegeben durch Cambridge University Press.

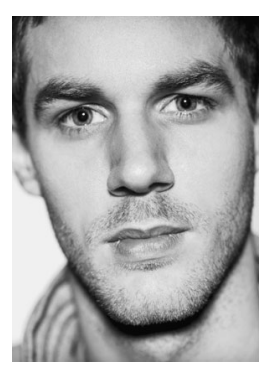

\section{Sebastian Wolfgang Sattler}

wurde 1988 in St. Pölten, Österreich, geboren. Er schloss 2015 sein Elektrotechnikstudium an der Technischen Universität Graz ab. Im Laufe des Studiums spezialisierte er sich in Richtung HF-Technik mit dem Schwerpunkt auf Messungen bei mm-Wellen-Frequenzen. Seit November 2015 ist er an der TU Graz am Institut für Hochfrequenztechnik als wissenschaftlicher Projektmitarbeiter angestellt und hat sein Doktoratsstudium im Februar 2016 begonnen. Der Schwerpunkt der Doktorarbeit liegt auf der Implementierung von planaren Strukturen bei mm-Wellen-Frequenzen.

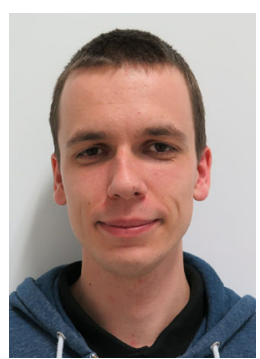

\section{Dominik Amschl}

wurde in Graz, Österreich, geboren. Nach seinem Abschluss an der HTL Bulme Graz/Gösting begann er sein Studium im Bereich Elektrotechnik an der Technischen Universität Graz. Momentan arbeitet er am Institut für Hochfrequenztechnik der TU Graz. Sein Aufgabenbereich beinhaltet unter anderem HF- und mm-Wellen-Messungen bis $110 \mathrm{GHz}$.

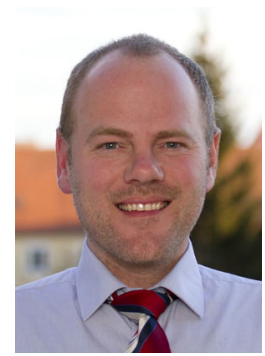

\section{Bernhard Auinger}

received the master's degree in electrical engineering (Dipl. Ing.) in 2004 and the Dr. techn. degree with distinction in 2015, both from Graz University of Technology, Austria. From 2011 to 2015 he was involved in theoretical investigations for wireless communications and on test procedures for MIMO enabled user equipment using LTE, which was a cooperation between the Institute of Microwave and Photonic Engineering, Graz University of Technology, and the company Rohde \& Schwarz, Munich, Germany. From 2005 to 2011 he initiated and led the electromagnetic compatibility group for automotive ICs at Philips Semiconductors and NXP Semiconductors. During his studies he was engaged in the comet mission ROMAP/ROSETTA of the European Space Agency (ESA). Currently, he is researcher at the Institute of Electronics, Graz University of Technology, and investigates new semiconductor technologies and methods for increasing the efficiency, shrinking the unit size and reducing emissions of welding machines and switched power converters for solar power, also applying wireless communication theory in power electronics.

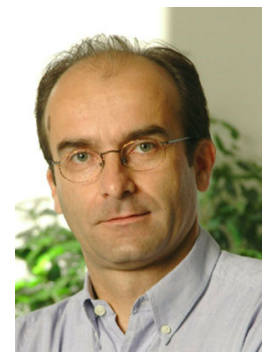

\section{Gerald Holweg}

absolvierte an der Technischen Universität Graz das Studium der Elektrotechnik und begann anschließend 1983 seine berufliche Laufbahn als ASIC-Entwicklungsingenieur bei AMI-Austria. 1987 wechselte er zur Startup Company MIKRON-Austria, wo er als Projektleiter unter anderem für die Entwicklung von RFID-Technologien und Produkten wie z. B. MIFARE $®$ verantwortlich war. 1995 wurde MIKRON-Austria in Philips Identification eingegliedert, wo Gerald Holweg als Entwicklungsleiter für Contactless Smart Cards tätig war. 1998 wechselte er zum Siemens-Entwicklungszentrum für Mikroelektronik, später Infineon Development Center Graz, das zu diesem Zeitpunkt einen neuen Standort in Graz eröffnete. Als Leiter der Produktentwicklung Chip Card und Security ICs war er verantwortlich für den Aufbau des neuen Teams und für die Entwicklung von CC, Security und RFID-Produkten. Seit 2005 ist Herr Holweg im Infineon DC Graz für Vorfeldentwicklung und industrielle Forschung verantwortlich.

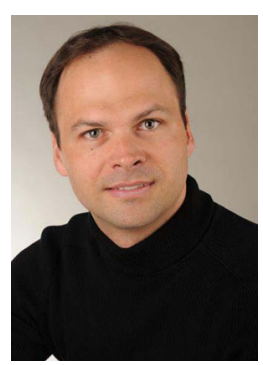

Ulrich Mühlmann

received his master's degree (Dipl.-Ing. in telematics) from the Graz University of Technology (TU Graz), Austria, in 2000. From 2000 to 2001 he attended the basic training course in the Austrian army. In 2001 he started research work in optical tracking at the Department of Electrical Measurement and Measurement Signal Processing (TU Graz). In November 2005 he received his Ph.D. degree (Dr. techn.) with honors. Since 2005 he has been with NXP Semiconductors (former Philips Semiconductors) working in the field of RFID/NFC technology focused on systems and analog innovation. He is author and/or co-author of several publications in the field of optical tracking, machine vision and RFID. 
Ulrich Mühlmann is a member of several standardization bodies. In ETSI he is significantly involved in several groups (TG28, TG34) in the field of RFID/NFC spectrum mask engineering and channel management. He is also a member of CEPT/ECC working in the field of RFID spectrum management.

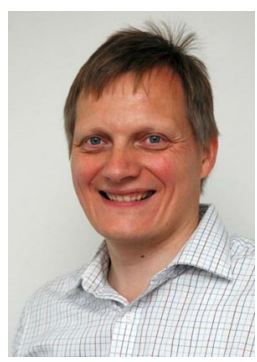

\section{Wolfgang Bösch}

ist seit März 2010 Leiter des neu eingerichteten Instituts für Hochfrequenztechnik an der Technischen Universität Graz, Österreich. Er schloss sein Diplomstudium im Bereich Nachrichtentechnik an der Technischen Universität in Wien, Österreich, im Jahr 1985 und sein Doktoratsstudium an der Technischen Universität in Graz jeweils mit Auszeichnung ab. 2004 absolvierte er den MBA mit Auszeichnung an der Bradford University School of Management. In den Jah- ren zuvor war er als CTO des Advanced Digital Institute in England tätig, bei RFMD (UK) als Direktor für "Business and Technology Integration" und mehr als zehn Jahre als CTO im Geschäftsbereich "Integrated Products" sowie als Direktor der "Global Technology Group" bei Filtronic plc in England beschäftigt. Vor seiner Tätigkeit bei Filtronic beschäftigte er sich bei der Europäischen Weltraumorganisation (ESA) im Bereich HF-Leistungsverstärker und deren Linearisierung bzw. mit intergierten Mikrowellenschaltungen und anderen Forschungsprojekten bei M/A-COM in Boston, MA, USA. Er war außerdem vier Jahre bei DaimlerChrysler Aerospace mit der Entwicklung von T/R-Modulen für luftgestützte Radare beschäftigt. Prof. Bösch ist Fellow des Institute of Electrical and Electronics Engineers (IEEE) und Fellow der Institution of Engineering and Technology (IET). Er publizierte mehr als 80 Papers und hält vier Patente. Er war als Non-Executive Director für Diamond Microwave Devices (DMD) und dem Advanced Digital Institute (ADI) tätig. Aktuell ist er Non-Executive Director für VIPER-RF (UK). 